\title{
MIR337 Pre-miRNA
}

National Cancer Institute

\section{Source}

National Cancer Institute. MIR337 Pre-miRNA. NCI Thesaurus. Code C82821.

MIR337 is an oligoribonucleotide that is encoded by the human MIR337 gene and has a role in the regulation of gene expression. 